\title{
Schädelperforationen Eingeborener vom Sepik in Neuguinea
}

Otto Schlaginhaufen

An einem Teil der menschlichen Schädel, die ich während der Fahrt auf dem Sepik-Fluß in der Zeit vom 30. Juli bis 6. August 1909 sammelte, sind Besonderheiten $\mathrm{zu}$ bemerken, die nicht in erster Linie als anthropologische, sondern als ethnographische Erscheinungen $\mathrm{zu}$ bewerten sind und daher wohl eine gesonderte Betrachtung für sich in Anspruch nehmen dürfen. Über die Reise wurde bei früheren Gelegenheiten schon ausführlich berichtet (Schlaginhaufen 1910-1911, 5-9; 1959, 174-186).

Die Schädelserie, die ich am Unterlauf des SepikFlusses - früher Kaiserin-Augusta-Fluß genannt - sammelte, umfaßt 48 Stück. Von ihnen sind es ihrer 22, welche eine Perforation der Schädeldecke aufweisen. Sie ist in den meisten Fällen dem Bregma-Punkt, das heißt jener Stelle benachbart, wo die Sutura sagittalis auf die Sutura coronalis trifft. Die transversale Ausdehnung der Durchbohrung

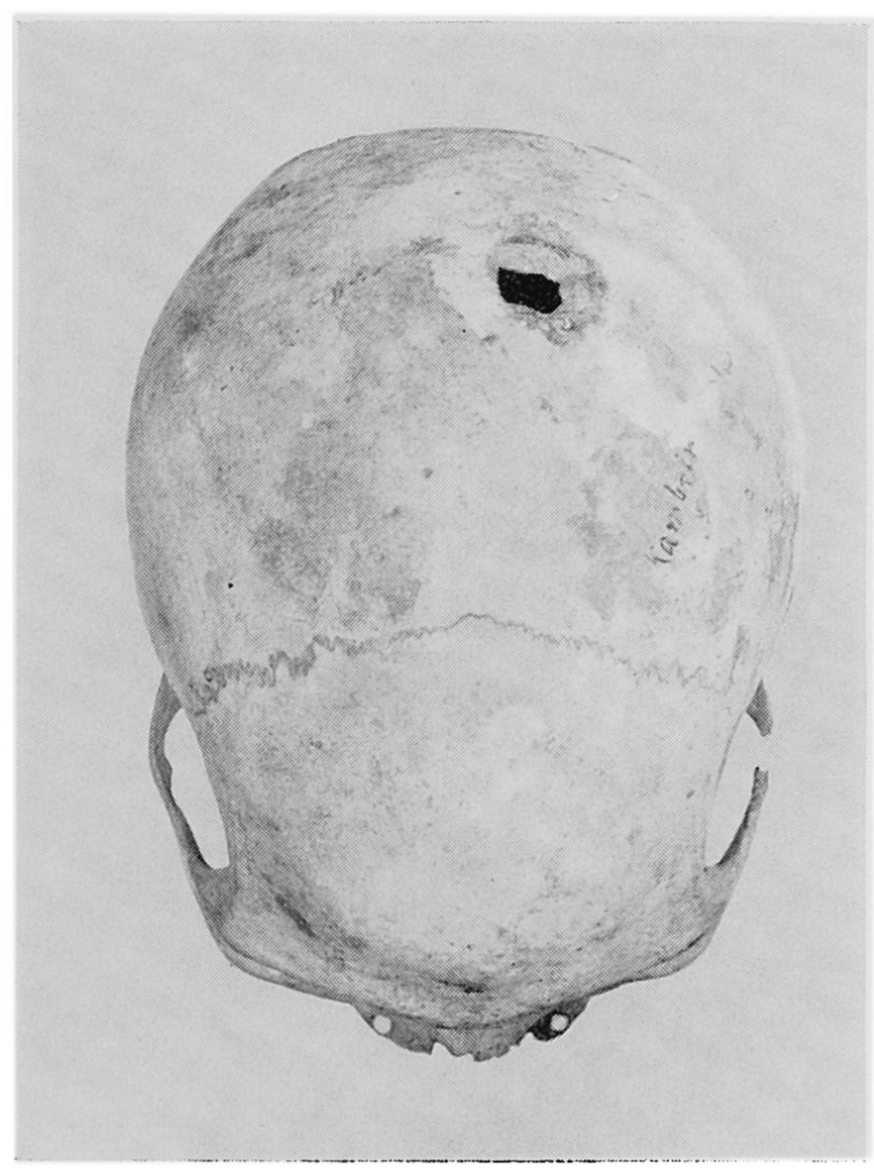

Abb. 1 (Nr. 4178)

Schädel vom Unterlauf des Sepik in Neuguinea variiert von 3 bis $26 \mathrm{~mm}$, die sagittale von 3 bis 23 $\mathrm{mm}$; das Verhältnis zwischen beiden Durchmessern, das bei 21 Schädeln von 38,5 bis 127,8 schwankt, beträgt im Durchschnitt 97,1. Das Überwiegen des transversalen Durchmessers ist jedoch $\mathrm{zu}$ gering, als daß daraus für die Bedeutung der Perforationen Schlüsse gezogen werden könnten.

Die Untersuchung der Lage der Perforation zu den drei Knochen, das heißt den beiden Ossa parietalia und dem Os frontale, ergab das Folgende: Weitaus am häufigsten fand sich die Perforation im Bereich der Ossa parietalia, und zwar 5mal rechts, $6 \mathrm{mal}$ links und $6 \mathrm{mal}$ auf beiden Seiten zugleich. In den einseitigen Fällen war in der Regel der medialen frontalen Ecke der Vorzug gegeben. Abweichungen von dieser Regel zeigen die Schädel Nr. 4178 und Nr. 4181 (Abb. 1 und 2). Die Entfernung des Lückenrandes von der Sutura coronalis beträgt im ersten Fall $66 \mathrm{~mm}$, im letzten $39 \mathrm{~mm}$. Ausschließlich

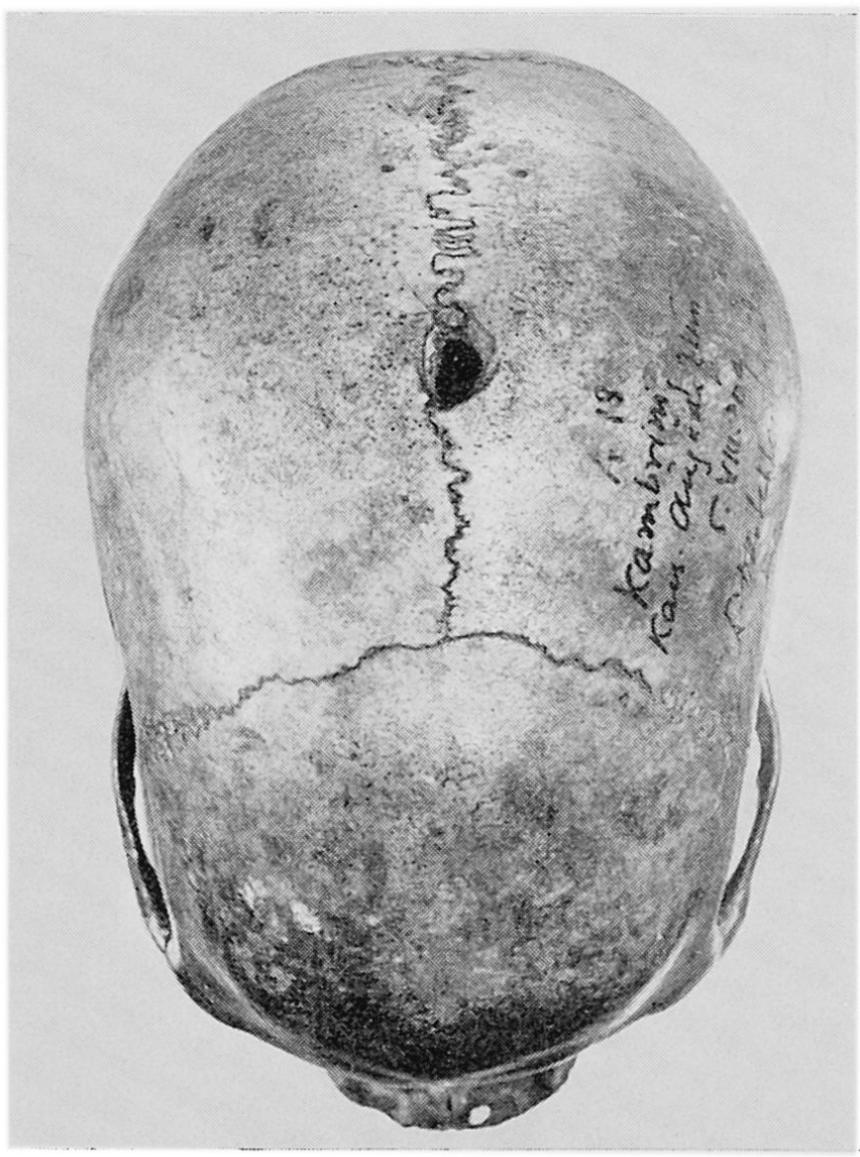

Abb. 2 (Nr. 4181) 


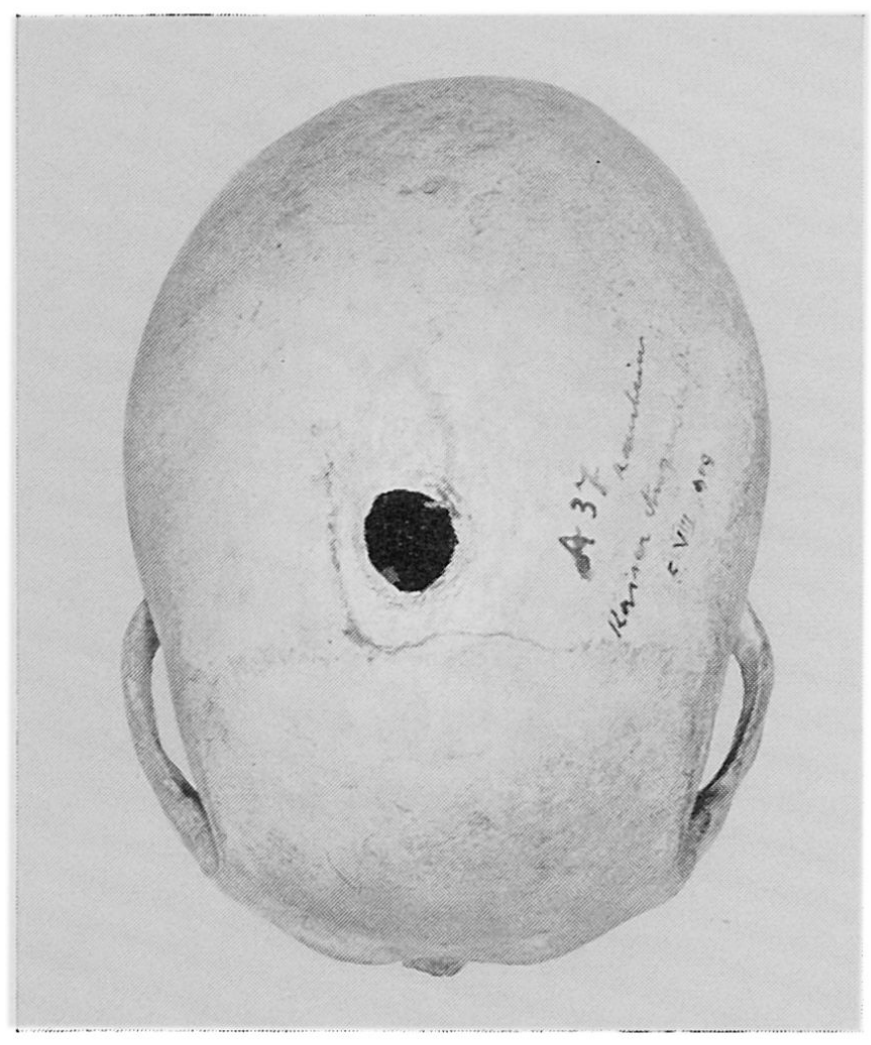

Abb. 3 (Nr. 4209)

Schädel vom Unterlauf des Sepik in Neuguinea

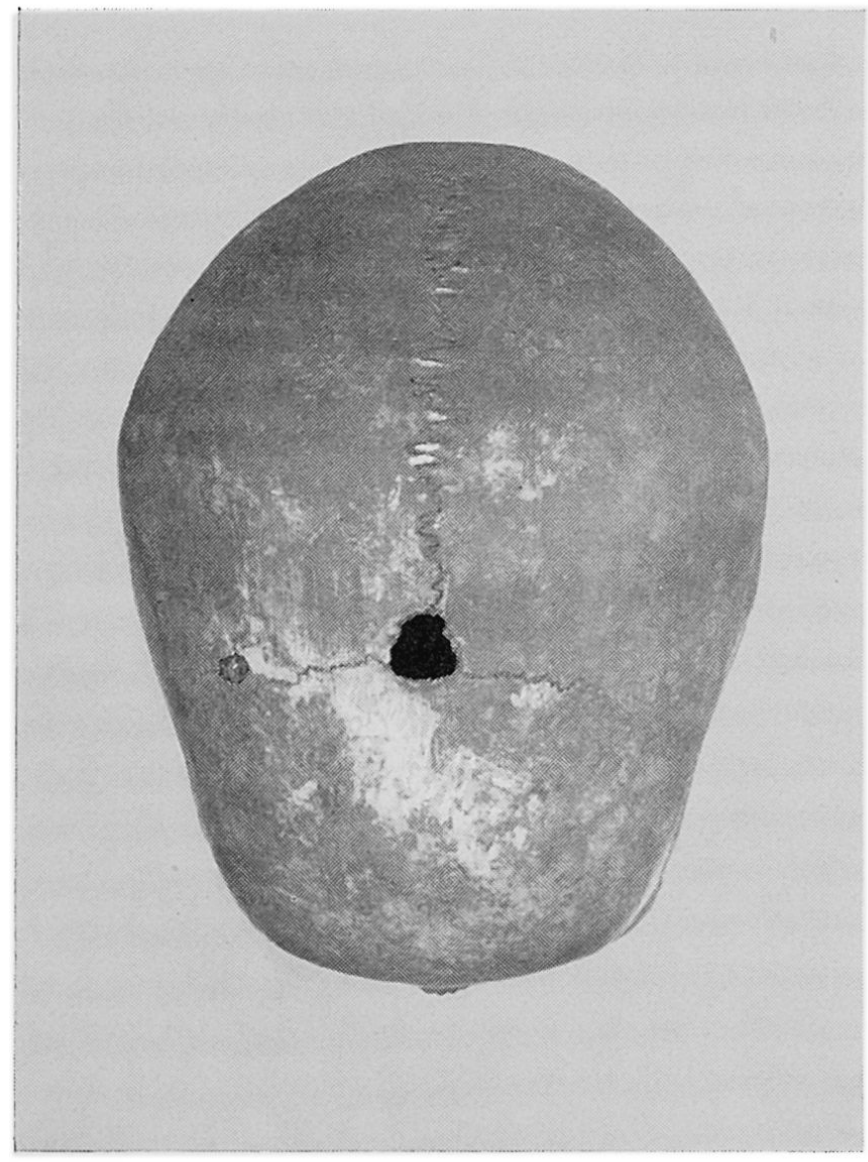

Abb. 5 (Nr. 4213)

Schädel vom Unterlauf des Sepik in Neuguinea

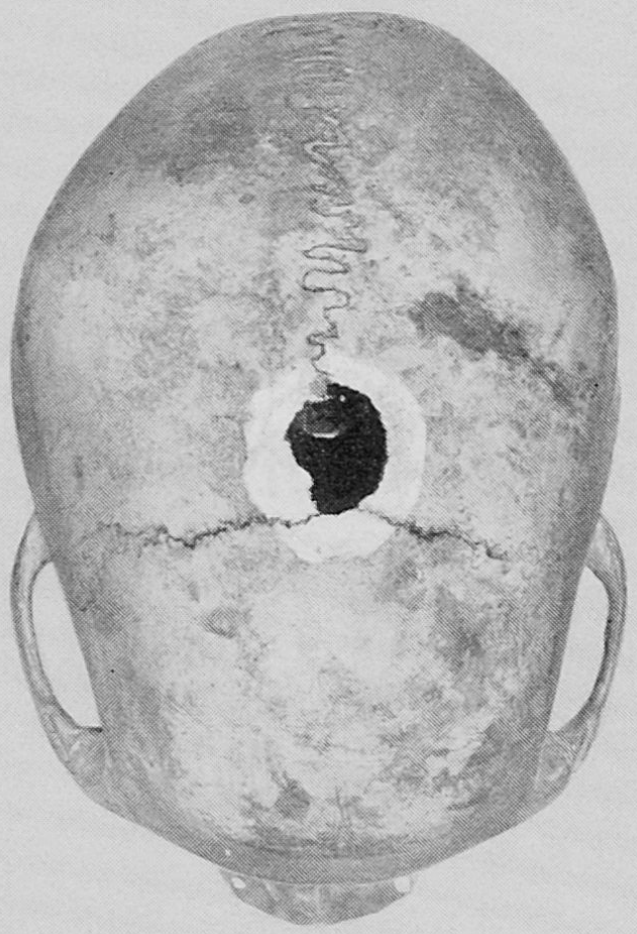

Abb. 4 (Nr. 4194)

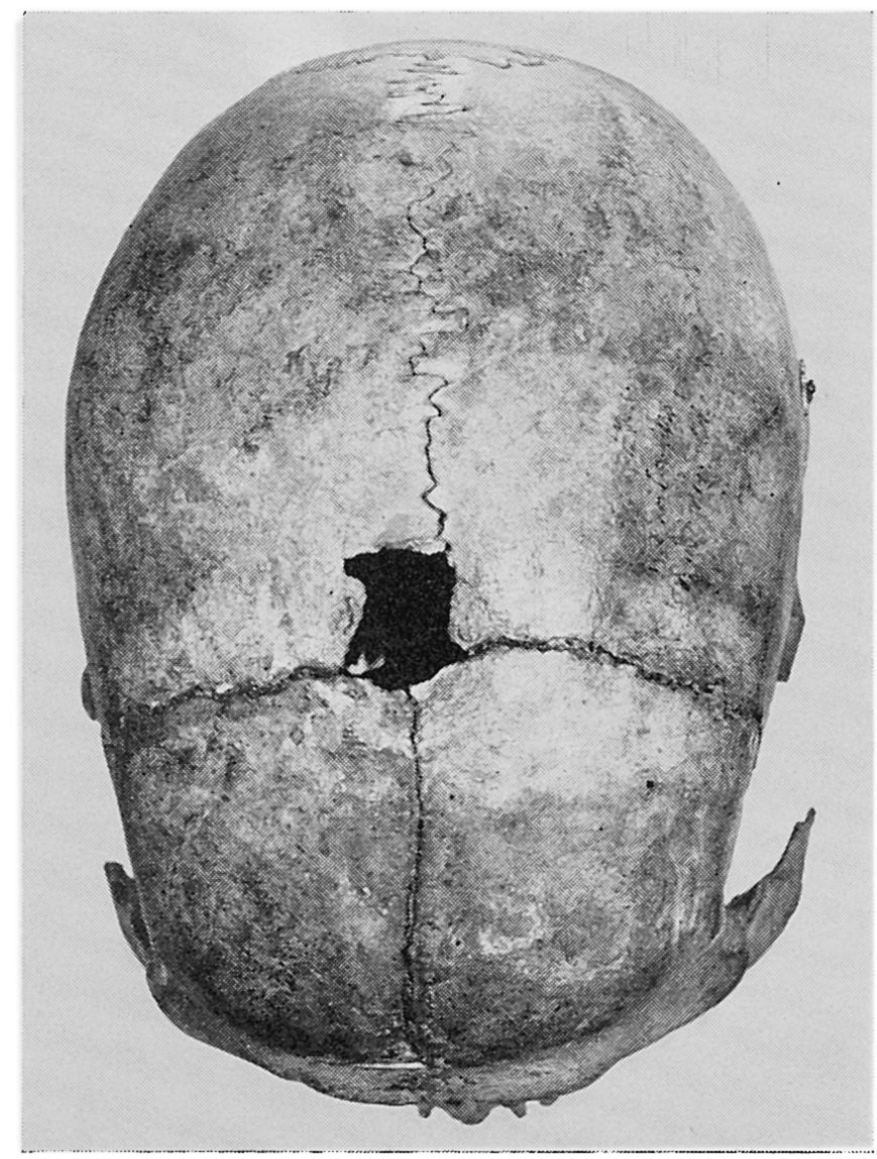

Abb. 6 (Nr. 4173) 


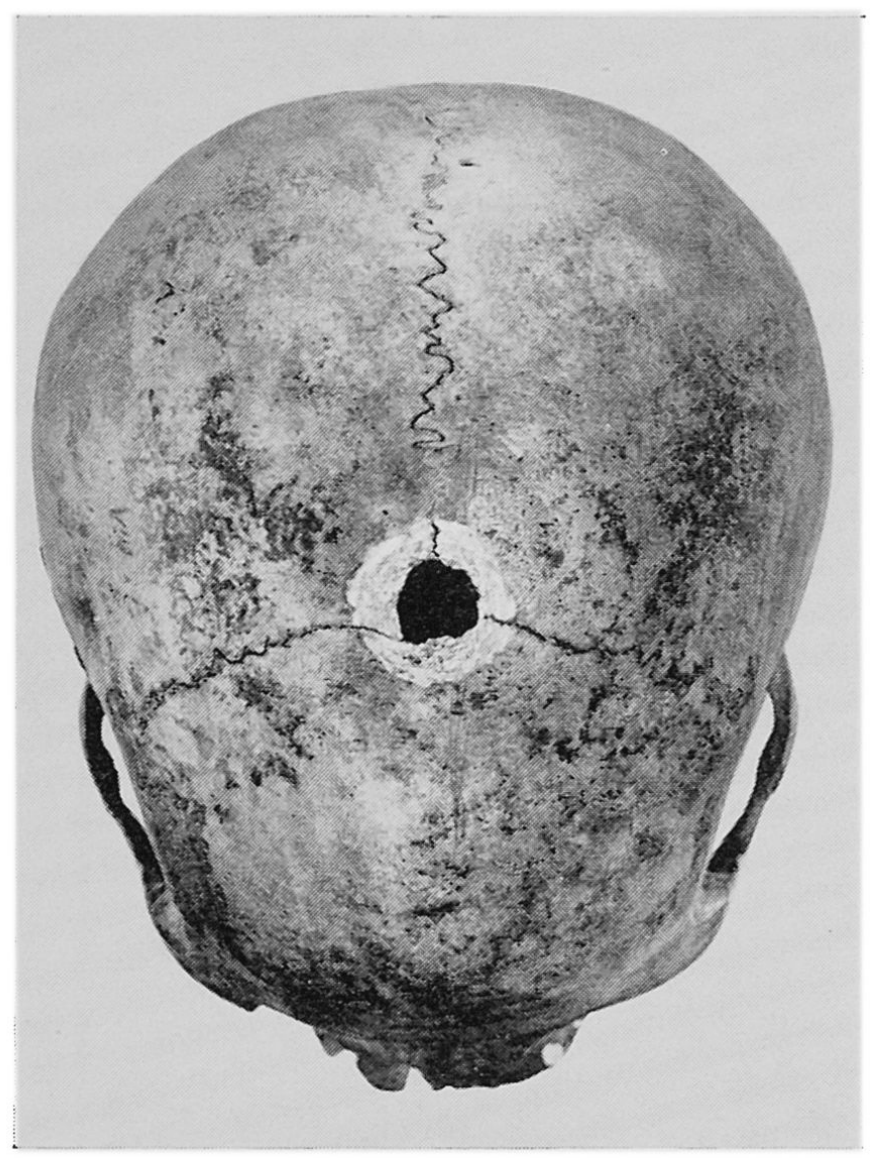

Abb. 7 (Nr. 4198)

Schädel vom Unterlauf des Sepik in Neuguinea

im Bereich des rechten Os parietale fand sich die Perforation bei Nr. 4209 (Abb. 3). Alle drei Knochen beteiligen sich gleichzeitig an derselben Perforation bei Nr. 4193 und Nr. 4194 (Abb. 4). Bei Schädel Nr. 4204 handelt es sich um eine $3 \mathrm{~mm}$ vor der Sutura coronalis befindliche und anscheinend sorgfältig ausgeführte Durchbohrung des Os frontale.

Die allgemeine Form der Perforationslücke, die einer exakt metrischen Darstellung wohl kaum zugänglich ist, läßt sich in sechs Kategorien ordnen, die in folgenden Frequenzen festzustellen waren: Die dreiseitige Form trat in 4 Fällen auf (Nr. 4213, Abb. 5), die vierseitige (Nr. 4173, Abb. 6) in 5, die Kreisform (Nr. 4198, Abb. 7) ebenfalls in 5, die Eiform in 3, die Birnform in 2 Fällen, und zweimal hatten die Perforationen lediglich den Charakter von engen Löchern (Nr. 4172, Abb. 8). Knochenplättchen, die vielleicht als vorübergehender Verschluß oder als Teil eines solchen hätten aufgefaßt werden können, wurden nicht beobachtet.

Den Bemühungen um die Erkenntnis der Perforationen der Schädeldecke war bis jetzt geringer $\mathrm{Er}$ folg beschieden. Zunächst wurde bei der einzelnen Perforation an ein Hilfsmittel bei einer Aufhängevorrichtung des Schädels gedacht. Der Knäuel einer Schnur wurde durch das Foramen magnum in den Innenraum des Schädels gebracht und das freie

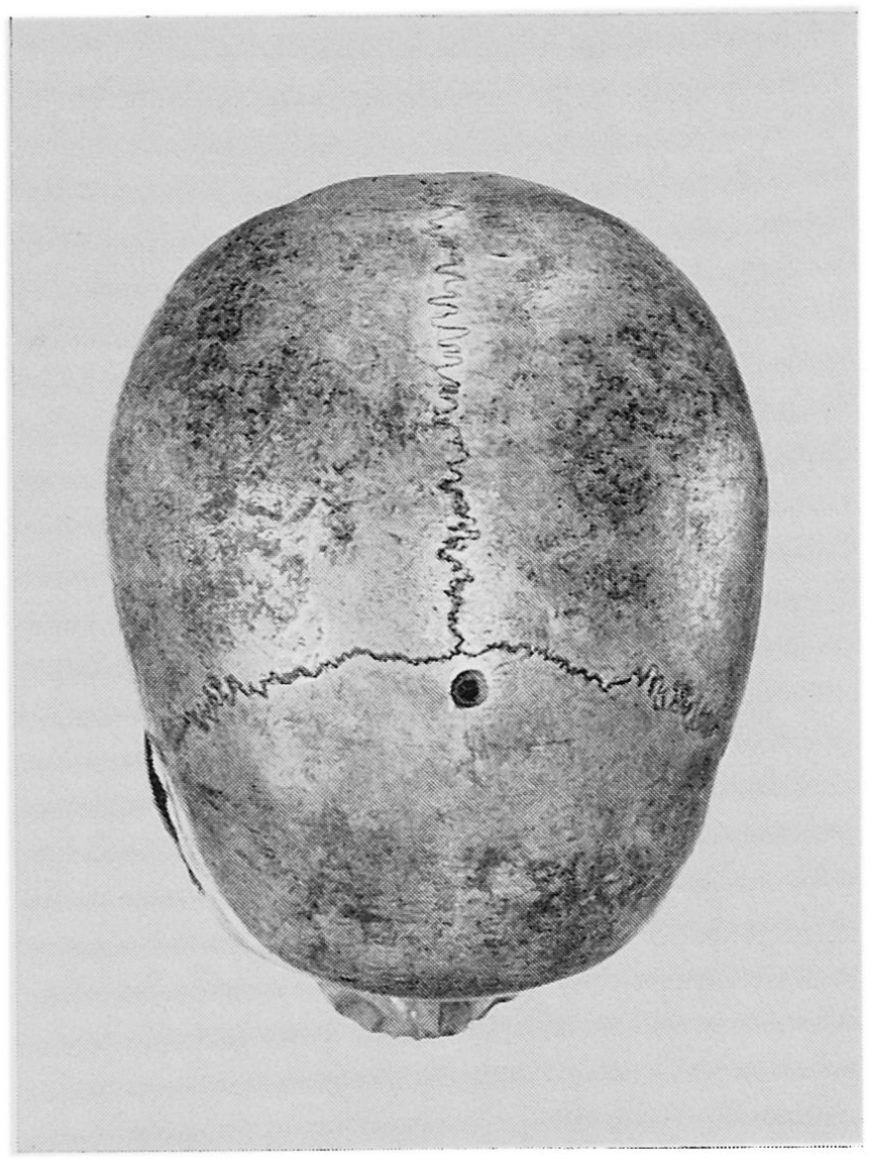

Abb. 8 (Nr. 4172)

Ende der Schnur durch die perforierte Lücke bis zu einer hochgelegenen Stelle, zum Beispiel am Dach oder einem Baum, geführt und dort befestigt. Für die Tatsache, daß für die Befestigung noch besondere Mittel, wie klebrige Substanzen, Verwendung fanden, spricht das gelegentliche Vorkommen anders getönter Flecken und Striche in der Umgebung der Perforationslücke.

Eine andere Auffassung geht lediglich dahin, daß von außen her in die Perforationslücke schmückende oder dem Kult dienende Dinge hineingesteckt wurden, ohne daß der Schädel aufgehängt war.

Auf welche Weise die Schädel befestigt waren, welche Fülleborn in einem Zeremonialhaus des 375$\mathrm{km}$-Dorfes photographisch aufgenommen und $R e$ che (1913, Taf. XXXVIII) publiziert hat, läßt das Bild der Schädel nicht erkennen.

Es muß späteren Forschungen überlassen bleiben, die Bedeutung der Perforationen zu ergründen.

\section{Literatur}

Reche, Otto, 1913: Der Kaiserin-Augusta-Fluß. Friederichsen und Co., Hamburg. - Schlaginhaufen, Otto, 1910-1911: Reisen in Kaiser-Wilhelmsland (Neuguinea). Abhandlungen und Berichte des k. Zool. und Anthrop.-Ethnograph. Museums zu Dresden, Bd. XIII, 1959. - Schlaginhaufen, Otto: Muliana: Zwei Jahre unter Südsee-Insulanern; Orell Füßli Verlag, Zürich. 